\title{
A NOTE ON STANDARD EQUIVALENCES
}

\author{
XIAO-WU CHEN
}

\begin{abstract}
We prove that any derived equivalence between triangular algebras is standard, that is, it is isomorphic to the derived tensor functor given by a two-sided tilting complex.
\end{abstract}

\section{INTRODUCTION}

Let $k$ be a field. We will require that all categories and functors are $k$-linear. Let $A$ be a finite dimensional $k$-algebra. We denote by $A$-mod the category of finite dimensional left $A$-modules and by $\mathbf{D}^{b}(A$-mod $)$ its bounded derived category.

Let $B$ be another finite dimensional $k$-algebra. We will require that $k$ acts centrally on any $B$-A-bimodule. Recall that a two-sided tilting complex is a bounded complex $X$ of $B$ - $A$-bimodules such that the derived tensor functor gives an equivalence $X \otimes_{A}^{\mathbf{L}}-: \mathbf{D}^{b}(A$-mod $) \rightarrow \mathbf{D}^{b}(B$-mod).

A triangle equivalence $F: \mathbf{D}^{b}(A$-mod $) \rightarrow \mathbf{D}^{b}(B$-mod $)$ is said to be standard if it is isomorphic, as a triangle functor, to $X \otimes_{A}^{\mathbf{L}}$ - for some two-sided tilting complex $X$. It is an open question whether all triangle equivalences are standard. The aim of this note is to answer this question affirmatively in a special case.

Recall that the algebra $A$ is triangular provided that the Ext-quiver of $A$ has no oriented cycles. There are explicit examples of algebras $A$ and $B$, which are derived equivalent such that $A$ is triangular, but $B$ is not; consult the top of [2, p.21]. It makes sense to have the following notion: an algebra $A$ is derived-triangular if it is derived equivalent to a triangular algebra.

Theorem 1.1. Let $A$ be a derived-triangular algebra. Then any triangle equivalence $F: \mathbf{D}^{b}(A$-mod $) \rightarrow \mathbf{D}^{b}(B$-mod $)$ is standard.

We observe that a derived-triangular algebra has finite global dimension. The converse is not true in general. Indeed, let $A$ be a non-triangular algebra with two simple modules which has finite global dimension. Then $A$ is not derived-triangular. Indeed, any triangular algebra $B$ which is derived equivalent to $A$ has two simple modules and thus is hereditary. This forces that the algebra $A$ is triangular, yielding a contradiction.

We recall that a piecewise hereditary algebra is triangular. In particular, Theorem 1.1 implies that the assumption on the standardness of the auto-equivalence in [3, Section 4] is superfluous.

The proof of Theorem 1.1 is a rather immediate application of [1, Theorem 4.7], which characterizes certain triangle functors between the bounded homotopy categories of Orlov categories. Here, we observe that the category of projective modules over a triangular algebra is naturally an Orlov category.

We refer to [4, 5] for unexplained notions in the representation theory of algebras.

Date: August 26, 2018.

2010 Mathematics Subject Classification. 13D09, 16G10, 18E30.

Key words and phrases. tilting complex, standard equivalence, Orlov category, homotopy category, triangle functor.

E-mail: xwchen@mail.ustc.edu.cn. 


\section{The Bounded homotopy CATEGORY OF AN ORLOV CATEGORY}

Let $\mathcal{A}$ be a $k$-linear additive category, which is Hom-finite and has split idempotents. Here, the Hom-finitess means that all the Hom spaces are finite dimensional. It follows that $\mathcal{A}$ is a Krull-Schmidt category.

We denote by Ind $\mathcal{A}$ a complete set of representatives of indecomposable objects in $\mathcal{A}$. The category $\mathcal{A}$ is called bricky if the endomorphism algebra of each indecomposable object is a division algebra.

We slightly generalize [1, Definition 4.1]. A bricky category $\mathcal{A}$ is called an Orlov category provided that there is a degree function $\operatorname{deg}:$ Ind $\mathcal{A} \rightarrow \mathbb{Z}$ with the following property: for any indecomposable objects $P, P^{\prime}$ having $\operatorname{Hom}_{\mathcal{A}}\left(P, P^{\prime}\right) \neq 0$, we have that $P \simeq P^{\prime}$ or $\operatorname{deg}(P)>\operatorname{deg}\left(P^{\prime}\right)$. An object $X$ in $\mathcal{A}$ is homogeneous of degree $n$, if it is isomorphic to a finite direct sum of indecomposables of degree $n$. An additive functor $F: \mathcal{A} \rightarrow \mathcal{A}$ is homogeneous if it sends homogeneous objects to homogenous objects and preserves their degrees.

Let $A$ be a finite dimensional $k$-algebra. We denote by $\left\{S_{1}, S_{2}, \cdots, S_{n}\right\}$ a complete set of representatives of simple $A$-modules. Denote by $P_{i}$ the projective cover of $S_{i}$. We recall that the Ext-quiver $Q_{A}$ of $A$ is defined as follows. The vertex set of $Q_{A}$ equals $\{1,2, \cdots, n\}$, and there is a unique arrow from $i$ to $j$ provided that $\operatorname{Ext}_{A}^{1}\left(S_{i}, S_{j}\right) \neq 0$. The algebra $A$ is triangular provided that $Q_{A}$ has no oriented cycles.

Let $A$ be a triangular algebra. We denote by $Q_{A}^{0}$ the set of sources in $Q_{A}$. Here, a vertex is a source if there is no arrow ending at it. For each $d \geq 1$, we define the set $Q_{A}^{d}$ inductively, such that a vertex $i$ belongs to $Q_{A}^{d}$ if and only if any arrow ending at $i$ necessarily starts at $\bigcup_{0 \leq m \leq d-1} Q_{A}^{m}$. It follows that $Q_{A}^{0} \subseteq Q_{A}^{1} \subseteq Q_{A}^{2} \subseteq \cdots$ and that $\bigcup_{d>0} Q_{A}^{d}=\{1,2, \cdots, n\}$. We mention that this construction can be found in [4, p.42].

We denote by $A$-proj the category of finite dimensional projective $A$-modules. Then $\left\{P_{1}, P_{2}, \cdots, P_{n}\right\}$ is a complete set of representatives of indecomposables in $A$-proj. For each $1 \leq i \leq n$, we define $\operatorname{deg}\left(P_{i}\right)=d$ such that $i \in Q_{A}^{d}$ and $i \notin Q_{A}^{d-1}$.

The following example of an Orlov category seems to be well known.

Lemma 2.1. Let $A$ be a triangular algebra. Then A-proj is an Orlov category with the above degree function. Moreover, any equivalence $F: A$-proj $\rightarrow A$-proj is homogeneous.

Proof. Since $A$ is triangular, it is well known that $\operatorname{End}_{A}\left(P_{i}\right)$ is isomorphic to $\operatorname{End}_{A}\left(S_{i}\right)$, which is a division algebra. Then $A$-proj is bricky. We recall that for $i \neq j$ with $\operatorname{Hom}_{A}\left(P_{i}, P_{j}\right) \neq 0$, there is a path from $j$ to $i$ in $Q_{A}$. From the very construction, we infer that for an arrow $\alpha: a \rightarrow b$ with $b \in Q_{A}^{d}$, we have $a \in Q_{A}^{d-1}$. Then we are done by the following consequence: if there is a path from $j$ to $i$ in $Q_{A}$, then $\operatorname{deg}\left(P_{j}\right)<\operatorname{deg}\left(P_{i}\right)$.

For the final statement, we observe that the equivalence $F$ extends to an autoequivalence on $A$-mod, and thus induces an automorphism of $Q_{A}$. The automorphism preserves the subsets $Q_{A}^{d}$. Consequently, the equivalence $F$ preserves degrees, and is homogeneous.

Let $\mathcal{A}$ be a $k$-linear additive category as above. We denote by $\mathbf{K}^{b}(\mathcal{A})$ the homotopy category of bounded complexes in $\mathcal{A}$. Here, a complex $X$ is visualized as $\cdots \rightarrow X^{n-1} \stackrel{d_{X}^{n-1}}{\rightarrow} X^{n} \stackrel{d_{X}^{n}}{\rightarrow} X^{n+1} \rightarrow \cdots$, where the differentials satisfy $d_{X}^{n} \circ d_{X}^{n-1}=0$. The translation functor on $\mathbf{K}^{b}(\mathcal{A})$ is denoted by [1], whose $n$-th power is denoted by $[n]$.

We view an object $A$ in $\mathcal{A}$ as a stalk complex concentrated at degree zero, which is still denoted by $A$. In this way, we identify $\mathcal{A}$ as a full subcategory of $\mathbf{K}^{b}(\mathcal{A})$. 
We are interested in triangle functors on $\mathbf{K}^{b}(\mathcal{A})$. We recall that a triangle functor $(F, \theta)$ consists of an additive functor $F: \mathbf{K}^{b}(\mathcal{A}) \rightarrow \mathbf{K}^{b}(\mathcal{A})$ and a natural isomorphism $\theta:[1] F \rightarrow F[1]$, which preserves triangles. More precisely, for any triangle $X \rightarrow Y \rightarrow Z \stackrel{h}{\rightarrow} X[1]$ in $\mathbf{K}^{b}(\mathcal{A})$, the sequence $F X \rightarrow F Y \rightarrow F Z \stackrel{\theta_{X} \circ F(h)}{\longrightarrow}(F X)[1]$ is a triangle. We refer to $\theta$ as the commutating isomorphism for $F$. A natural transformation between triangle functors is required to respect the two commutating isomorphisms.

For a triangle functor $(F, \theta)$, the commutating isomorphism $\theta$ is trivial if $[1] F=$ $F[1]$ and $\theta$ is the identity transformation. In this case, we suppress $\theta$ and write $F$ for the triangle functor.

Any additive functor $F: \mathcal{A} \rightarrow \mathcal{A}$ gives rise to a triangle functor $\mathbf{K}^{b}(F): \mathbf{K}^{b}(\mathcal{A}) \rightarrow$ $\mathbf{K}^{b}(\mathcal{A})$, which acts on complexes componentwise. The commuting isomorphism for $\mathbf{K}^{b}(F)$ is trivial. Similarly, any natural transformation $\eta: F \rightarrow F^{\prime}$ extends to a natural transformation $\mathbf{K}^{b}(\eta): \mathbf{K}^{b}(F) \rightarrow \mathbf{K}^{b}\left(F^{\prime}\right)$ between triangle functors.

The following fundamental result is due to [1, Theorem 4.7].

Proposition 2.2. Let $\mathcal{A}$ be an Orlov category, and let $(F, \theta): \mathbf{K}^{b}(\mathcal{A}) \rightarrow \mathbf{K}^{b}(\mathcal{A})$ be a triangle functor such that $F(\mathcal{A}) \subseteq \mathcal{A}$. We assume further that $\left.F\right|_{\mathcal{A}}: \mathcal{A} \rightarrow \mathcal{A}$ is homogeneous. Let $F_{1}, F_{2}: \mathcal{A} \rightarrow \mathcal{A}$ be two homogeneous functors.

(1) There is a unique natural isomorphism $(F, \theta) \rightarrow \mathbf{K}^{b}\left(\left.F\right|_{\mathcal{A}}\right)$ of triangle functors, which is the identity on the full subcategory $\mathcal{A}$.

(2) Any natural transformation $\mathbf{K}^{b}\left(F_{1}\right) \rightarrow \mathbf{K}^{b}\left(F_{2}\right)$ of triangle functors is of the form $\mathbf{K}^{b}(\eta)$ for a unique natural transformation $\eta: F_{1} \rightarrow F_{2}$.

Proof. The existence of the natural isomorphism in (1) is due to [1, Theorem 4.7]; compare [1, Remark 4.8]. The uniqueness follows from the commutative diagram (4.10) and Lemma 4.5(2) in 1], by induction on the support of a complex in the sense of [1, Subsection 4.1]. Here, we emphasize that the commutating isomorphism $\theta$ is used in the construction of the natural isomorphism on stalk complexes; compare the second paragraph in [1, p.1541].

The statement (2) follows from the uniqueness part of (1).

Recall that $\mathbf{D}^{b}(A$-mod) denotes the bounded derived category of $A$-mod. We identify $A$-mod as the full subcategory of $\mathbf{D}^{b}(A$-mod) formed by stalk complexes concentrated at degree zero. We denote by $H^{n}(X)$ the $n$-th cohomology of a complex $X$.

The following observation is immediate.

Lemma 2.3. Let $A$ be a finite dimensional algebra, and let $F: \mathbf{D}^{b}(A$-mod $) \rightarrow$ $\mathbf{D}^{b}(A$-mod $)$ be a triangle equivalence with $F(A) \simeq A$. Then we have $F(A$-mod $)=$ $A$-mod, and thus the restricted equivalence $\left.F\right|_{A \text {-mod }}: A$-mod $\rightarrow A$-mod.

Proof. We use the canonical isomorphisms $H^{n}(X) \simeq \operatorname{Hom}_{\mathbf{D}^{b}(A-\bmod )}(A[-n], X)$. It follows that both $F$ and its quasi-inverse send stalk complexes to stalk complexes. Then we are done.

We assume that we are given an equivalence $F: A$-mod $\rightarrow A$-mod with $F(A) \simeq$ $A$. Then there is an algebra automorphism $\sigma: A \rightarrow A$ such that $F$ is isomorphic to ${ }_{\sigma} A_{1} \otimes_{A}-$. Here, the $A$-bimodule ${ }_{\sigma} A_{1}$ is given by the regular right $A$-module, where the left $A$-module is twisted by $\sigma$. This bimodule is invertible and thus viewed as a two-sided tilting complex. We refer to [5, Section 6.5] for details on two-sided tilting complexes and standard equivalences.

We now combine the above results. 
Proposition 2.4. Let $A$ be a triangular algebra, and let $(F, \theta): \mathbf{D}^{b}(A$-mod $) \rightarrow$ $\mathbf{D}^{b}(A$-mod $)$ be a triangle equivalence with $F(A) \simeq A$. We recall the algebra automorphism $\sigma$ given by the restricted equivalence $\left.F\right|_{A \text {-mod }}$, and the $A$-bimodule ${ }_{\sigma} A_{1}$. Then there is a natural isomorphism $(F, \theta) \rightarrow{ }_{\sigma} A_{1} \otimes_{A}^{\mathbf{L}}-$ of triangle functors. In particular, the triangle equivalence $(F, \theta)$ is standard.

Proof. Since the algebra $A$ is triangular, it has finite global dimension. The natural functor $\mathbf{K}^{b}(A$-proj $) \rightarrow \mathbf{D}^{b}(A$-mod $)$ is a triangle equivalence. We identify these two categories. Therefore, the triangle functor $(F, \theta): \mathbf{K}^{b}(A$-proj $) \rightarrow \mathbf{K}^{b}(A$-proj) restricts to an equivalence $\left.F\right|_{A \text {-proj }}$, which is isomorphic to ${ }_{\sigma} A_{1} \otimes_{A}-$. By Lemma 2.1. the statements in Proposition 2.2 apply in our situation. Consequently, we have an isomorphism between $(F, \theta)$ and $\mathbf{K}^{b}\left({ }_{\sigma} A_{1} \otimes_{A}-\right)$. Then we are done.

\section{The Proof of Theorem 1.1}

We now prove Theorem 1.1. In what follows, for simplicity, when writing a triangle functor we suppress its commutating isomorphism.

We first assume that the algebra $A$ is triangular. The complex $F(A)$ is a onesided tilting complex. By [5, Theorem 6.4.1], there is a two-sided tilting complex $X$ of $B$ - $A$-bimodules with an isomorphism $X \rightarrow F(A)$ in $\mathbf{D}^{b}(B$-mod). Denote by $G$ a quasi-inverse of the standard equivalence $X \otimes_{A}^{\mathbf{L}}-: \mathbf{D}^{b}(A$-mod $) \rightarrow \mathbf{D}^{b}(B$-mod $)$. Then the triangle functor $G F: \mathbf{D}^{b}(A$-mod $) \rightarrow \mathbf{D}^{b}(A$-mod $)$ satisfies $G F(A) \simeq A$. Proposition 2.4 implies that $G F$ is standard, and thus $F$ is isomorphic to the composition of $X \otimes_{A}^{\mathbf{L}}$ - and a standard equivalence. Then we are done in this case by the well-known fact that the composition of two standard equivalences is standard.

In general, let $A$ be derived-triangular. Assume that $A^{\prime}$ is a triangular algebra which is derived equivalent to $A$. By [5, Proposition 6.5.5], there is a standard equivalence $F^{\prime}: \mathbf{D}^{b}\left(A^{\prime}\right.$-mod $) \rightarrow \mathbf{D}^{b}(A$-mod $)$. The above argument implies that the composition $F F^{\prime}$ is standard. Recall from [5, Proposition 6.5.6] that a quasiinverse $F^{\prime-1}$ of $F^{\prime}$ is standard. We are done by observing that $F$ is isomorphic to the composition $\left(F F^{\prime}\right) F^{\prime-1}$, a composition of two standard equivalences.

Acknowledgements We thank Martin Kalck for pointing out the example in 2. The author is supported by National Natural Science Foundation of China (No. 11201446), NCET-12-0507, and the Fundamental Research Funds for the Central Universities.

\section{REFERENCES}

[1] P.N. ACHAR, And S. Riche, Koszul duality and semisimplicity of Frobenius, Ann. Inst. Fourier 63(4) (2013), 1511-1612.

[2] G. Bonbinski, C. Geiss, And A. Skowronski, Classification of derived discrete algebras, Cent. Euro. J. Math. 2(1) (2004), 19-49.

[3] B. Keller, On triangulated orbit categories, Doc. Math. 10 (2005), 551-581.

[4] C.M. Ringel, Tame Algebras and Integral Quadratic Forms, Lect. Notes Math. 1099, Springer-Verlag, Berlin Heidelberg New York Tokyo, 1984.

[5] A. Zimmermann, Representation Theory, A Homological Algebra Point of View, Springer, International Publishing Switzerland, 2014.

Xiao-Wu Chen

Key Laboratory of Wu Wen-Tsun Mathematics, Chinese Academy of Sciences

School of Mathematical Sciences, University of Science and Technology of China

No. 96 Jinzhai Road, Hefei, 230026, Anhui, P.R. China.

URL: http://home.ustc.edu.cn/ ${ }^{\sim}$ xwchen 